\title{
SALUBRIDADE AMBIENTAL DO MUNICIPIO DE SÃO PEDRO DO IGUAÇU - PR
}

\author{
Luana Patrícia Pinto ${ }^{2}$, Ana Claudia $\mathrm{Cabral}^{3}$, Samara Moreira Perissato ${ }^{4}$, Késia Damaris de \\ Azevedo $^{5}$, Jianice Pires Frigo ${ }^{6}$, Elisandro Pires Frigo ${ }^{7}$
}

\footnotetext{
${ }^{1}$ Aceito para publicação no $1^{\circ}$ trimestre de 2014

${ }^{2}$ Acadêmica em Ciências Biológicas pela Universidade Federal do Paraná, Setor Palotina/PR, Brasil, luana.kozak@gmail.com;

${ }^{3}$ Universidade Estadual do Oeste do Paraná - UNIOESTE, mestrado em energia na agricultura, Rua Universitária n 2069, 85819-110, Cascavel - PR, ea.anaclaudia@gmail.com;

${ }^{4}$ Acadêmica em Agronomia pela Universidade Federal do Paraná, Setor Palotina/PR, Brasil, samaraperissato@gmail.com;

${ }^{5}$ Graduada em Tecnologia em Biotecnologia na Universidade Federal do Paraná, Palotina - PR, Brasil, kesia.damaris@gmail.com;

${ }^{6}$ Acadêmica em Agronomia pela Universidade Regional Integral do Alto Uruguai e das Missões - URI Campos de Santiago,jifrigo@yahoo.com.br;

${ }^{7}$ Prof. Dr. Adjunto da Universidade Federal do Paraná UFPR, Palotina - PR, Brasil, epfrigo@ gmail.com.
}

\section{RESUMO}

Salubridade ambiental é conhecida como a capacidade de prevenir a ocorrência de doenças ocasionadas pelo meio ambiente e promover o melhoramento da saúde pública e ecossistema. Inserido neste conceito há o Indicador de Salubridade Ambiental - ISA que é uma das maneiras de mensuração do estado em que se encontram determinados setores, do município, este baseiase em cinco tipos de indicadores: abastecimento de água, esgoto sanitário, resíduos sólidos, controles de vetores e recursos hídricos. No presente trabalho verificou-se a Salubridade do município de São Pedro do Iguaçu - PR, o qual apresentou ISA no valor de 79,58 sendo considerado salubre.

Palavras-chave: Mensuração, Qualidade, Desenvolvimento. 


\title{
ENVIRONMENTAL HEALTH OF THE MUNICIPALITY OF SAN PEDRO FALLS -
} PR

\begin{abstract}
Environmental health is known as the ability to prevent the occurrence of diseases caused by the environment and promote the public health and ecosystem improvement. The concept of Indicator of Environmental Health - ISA representes a way to measure the state of certain sectors of the city, this concept is based on five types of indicators: water supply, sewage, solid waste, vector control and water resources. In this stdy we verified the Environmental Health of the municipality of São Pedro do Igua, State of Paraná, which presented the ISA value 79.58, anda was considered wholesome.
\end{abstract}

Keywords: Measurement, quality, development.

\section{INTRODUÇÃO}

A preocupação com o saneamento ambiental brasileiro iniciou-se na década de 1970, embora a responsabilidade fosse dos municípios os serviços de saneamento eram executados por outros órgãos (FIOROTTI, 2008). Quando a qualidade de vida é analisada, deve-se relacionar bem-estar social, físico e mental sendo imprescindível tocar neste assunto.

Saneamento ambiental engloba o conjunto de ações socioeconômicas que visam alcançar um índice ideal de salubridade ambiental considerando alguns indicadores como o abastecimento de água potável local, coleta e disposição de resíduos poluentes, controle de doenças transmissíveis, drenagem urbana, e demais serviços (THAME, 1999).

De acordo com Thame (1999), salubridade ambiental remete ao estado de higidez da população urbana e rural, abrangendo a capacidade da prevenção de doenças, e promoção do bem estar local. É fundamental a listagem dos componentes influenciáveis no nível de salubridade da região. (JUNIOR, 2010).

Atualmente uma das formas de mensuração dos impactos ambientais causados pelo homem é a utilização dos indicadores ambientais aplicados nos conceitos de saneamento ambiental e de salubridade ambiental. No presente estudo foi utilizado o Indicador de Salubridade Ambiental - ISA, um modelo desenvolvido pela câmara técnica de planejamento do conselho estadual de Saneamento no Estado de São Paulo (CONESAN). Este sistema foi criado com a finalidade de avaliar a eficácia do plano estadual de saneamento, assim 
regulamentado pela Lei $\mathrm{n}^{\circ}$ 7.750, de 31 de março de 1992, a qual dispõe de algumas normas que buscam disciplinar metas e planejamentos de ações, obras e serviços no estado, respeitando a autonomia de cada município. (PIZA, 2000)

Esse índice é aplicado ao conceito estipulado acima de salubridade e que tem como objetivo verificar as condições ambientais em âmbito municipal, identificando e avaliando assim, as condições de saneamento de cada município (BATISTA, 2005).

O objetivo deste estudo foi analisar o estado da salubridade ambiental do município de São Pedro do Iguaçu - Paraná e avaliar a qualidade dos serviços públicos de saneamento da cidade.

\section{MATERIAL E MÉTODOS}

O presente trabalho baseia-se no ISA como forma de verificar os índices de salubridade promovidos pela ação humana. O ISA foi desenvolvido como instrumento na busca de indicadores de salubridade já que sintetiza as medidas a serem postas em práticas na obtenção de melhorias na qualidade de vida, incluindo-se os aspectos econômicos, sociais e de saúde pública para um desenvolvimento sustentável do município (BATISTA, 2005).

Para Piza (2000), o ISA possui um índice que é composto por indicadores da área da saúde pública, saneamento ambiental, recursos hídricos e socioeconômico. Estes são formados por cinco diferentes indicadores da água que engloba a quantidade de domicílios atendidos e a monitoração da qualidade de água fornecida; esgoto ao qual compara a oferta e demanda das instalações presentes e planejamento de futuras, como também a quantificação das residências atendidas com fossas sépticas ou redes; resíduos no qual se enfatiza a sua disposição final, e também a quantidade de domicílios coletados; vetores, dando um enfoque maior a doenças que predominam com a ineficácia do sistema de saúde pública; e, por fim os recursos hídricos o qual calculou o uso da água bruta ou risco geográfico, a disponibilidade de água nos mananciais e o controle da água em relação à distribuição municipal. Abaixo segue a equação que é utilizada para se calcular o ISA utilizando os critérios descritos acima:

Equação 1: Forma de cálculo dos indicadores ambientais

$\mathrm{ISA}=\left(0,26 \cdot \mathrm{I}_{\mathrm{AB}}\right)+\left(0,26 \cdot \mathrm{I}_{\mathrm{ES}}\right)+\left(0,26 \cdot \mathrm{I}_{\mathrm{RS}}\right)+\left(0,11 \cdot \mathrm{I}_{\mathrm{CV}}\right)+\left(0,11 \cdot \mathrm{I}_{\mathrm{RH}}\right)$

Onde:

Indicador de abastecimento de Água $\left(\mathrm{I}_{\mathrm{AB}}\right)$;

Indicador de Esgoto Sanitário (IES); 
Indicador de Resíduos Sólidos ( $\left.\mathrm{I}_{\mathrm{RS}}\right)$;

Indicador de Controle de Vetores $\left(\mathrm{I}_{\mathrm{CV}}\right)$;

Indicador de Riscos de Recursos Hídricos $\left(\mathrm{I}_{\mathrm{RH}}\right)$.

A Tabela 1 apresenta os componentes e sub indicadores que compõem o ISA:

Tabela 1: Sub indicadores e finalidades no ISA.

\begin{tabular}{|c|c|c|}
\hline \multirow[t]{2}{*}{ Componentes } & Sub indicadores & Finalidade \\
\hline & $\begin{array}{l}\text { Cobertura de } \\
\text { atendimento }\left(\mathrm{I}_{\mathrm{CA}}\right)\end{array}$ & $\begin{array}{l}\text { Quantificar os domicílios atendidos por } \\
\text { sistemas de abastecimento de água com } \\
\text { controle sanitário. }\end{array}$ \\
\hline \multirow{3}{*}{$\begin{array}{c}\text { Indicador de } \\
\text { abastecimento de } \\
\text { água }\left(\mathrm{I}_{\mathrm{AB}}\right)\end{array}$} & $\begin{array}{l}\text { Qualidade da água } \\
\left.\text { distribuída ( } \mathrm{I}_{\mathrm{QA}}\right)\end{array}$ & Monitorara a qualidade de água fornecida. \\
\hline & $\begin{array}{l}\text { Saturação do } \\
\text { sistema produtor } \\
\left.\text { (quantidade) (I } \mathrm{I}_{\mathrm{SA}}\right)\end{array}$ & $\begin{array}{l}\text { Comparar a oferta e a demanda de água; } \\
\text { programar ampliações ou novos sistemas } \\
\text { produtores e programas de controle e } \\
\text { redução de perdas. }\end{array}$ \\
\hline & $\begin{array}{c}\text { Cobertura em } \\
\text { coleta de esgotos e } \\
\text { tanques sépticos } \\
\left(\mathrm{I}_{\mathrm{CE}}\right)\end{array}$ & $\begin{array}{l}\text { Quantificar os domicílios atendidos por } \\
\text { redes de esgoto e/ou tanques sépticos. }\end{array}$ \\
\hline \multicolumn{3}{|l|}{ Indicador de } \\
\hline \multirow[t]{2}{*}{$\begin{array}{l}\text { esgoto sanitário } \\
\left.\text { (I } \mathrm{I}_{\mathrm{ES}}\right)\end{array}$} & $\begin{array}{l}\text { Esgoto tratado e } \\
\text { tanques sépticos } \\
\left.\text { ( } \mathrm{I}_{\mathrm{TE}}\right)\end{array}$ & Indicar a redução da carga poluidora. \\
\hline & $\begin{array}{l}\text { Saturação do } \\
\text { tratamento }\left(\mathrm{I}_{\mathrm{SE}}\right)\end{array}$ & $\begin{array}{l}\text { Comparar a oferta e demanda das } \\
\text { instalações existentes e programar novas } \\
\text { instalações ou ampliações. }\end{array}$ \\
\hline
\end{tabular}


Quantificar os domicílios atendidos por

Coleta de lixo $\left(\mathrm{I}_{\mathrm{CR}}\right)$ coleta de lixo.

\author{
Indicador de \\ resíduos sólidos \\ disposição final dos resíduos. \\ $\left(\mathrm{I}_{\mathrm{QR}}\right)$ \\ $\left(\mathrm{I}_{\mathrm{RS}}\right)$
}

Tratamento e Quantificar a situação da disposição final

Saturação da Indicar necessidade de novas instalações. deposição final

$\left(\mathrm{I}_{\mathrm{SR}}\right)$

Indicador de

controle de

vetores $\left(\mathrm{I}_{\mathrm{CV}}\right)$

Identificar a necessidade de programas

Dengue $\left(\mathrm{I}_{\mathrm{VD}}\right)$ e corretivos e preventivos de redução e

esquistossomose eliminação de vetores transmissores, e/ou

(IvE) hospedeiros da doença.

Indicar a necessidade de programas

Leptospirose ( $\left.\mathrm{I}_{\mathrm{VL}}\right)$ preventivos de redução e eliminação de ratos.

Quantificar a situação da água bruta ou Água bruta $\left(\mathrm{I}_{\mathrm{QB}}\right)$ risco geográfico.

Disponibilidade Quantificar a disponibilidade dos

dos mananciais mananciais em reação a demanda.

Indicador de $\quad\left(\mathrm{I}_{\mathrm{DM}}\right)$

recursos hídricos

$\left(\mathrm{I}_{\mathrm{RH}}\right)$

Abrange o controle das águas utilizadas

Fontes isoladas pelas populações em áreas urbanas não

( $\mathrm{I}_{\mathrm{FI}}$ atendidas pelos serviços oficiais de abastecimento de água. 


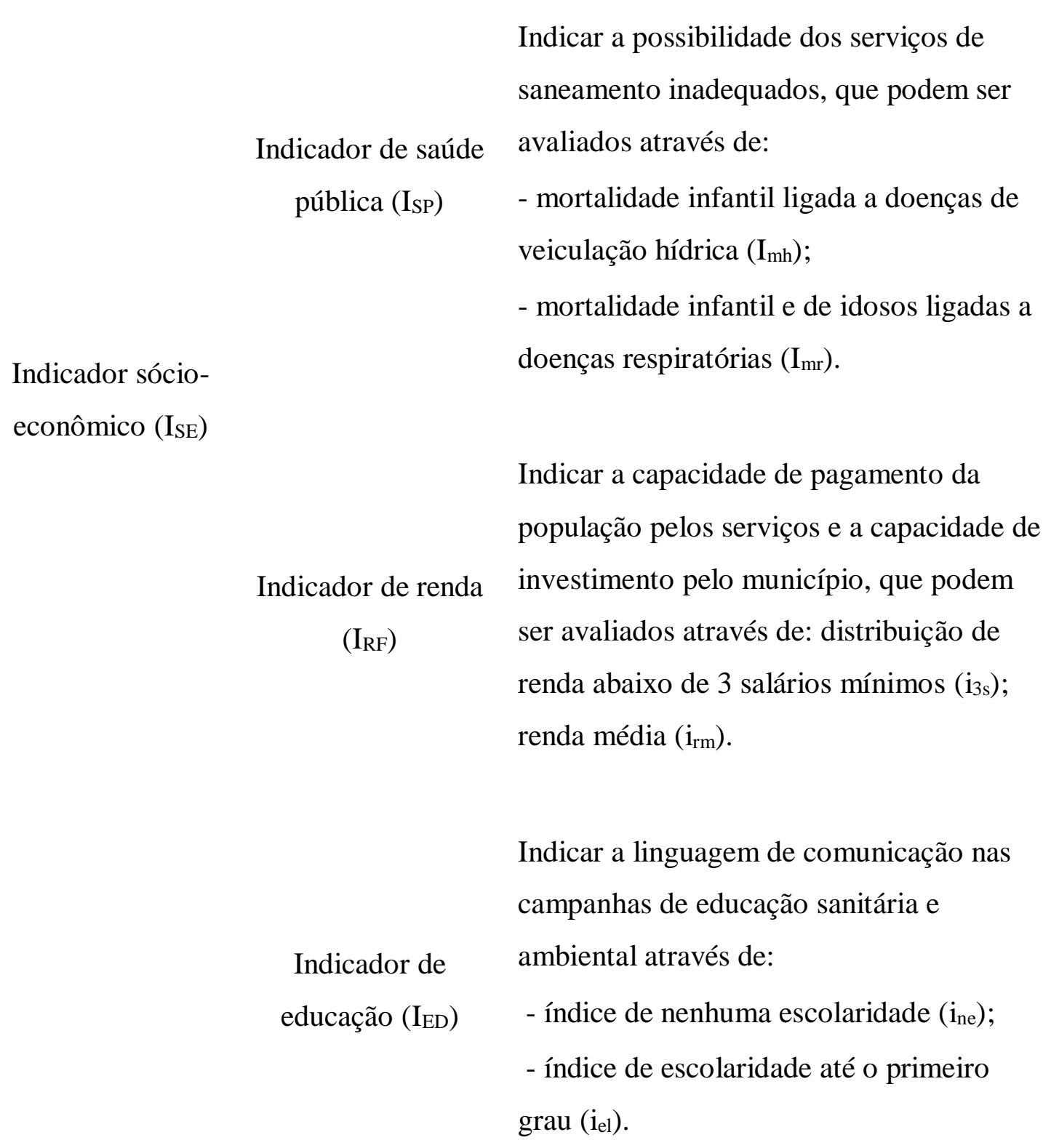

Fonte: BAHIA, 2006.

Baseado em Batista (2005), a seguinte tabela expõe a maneira que é feita a análise através do método ISA, seguindo uma classificação qualitativa, podendo-se assim, escolher e ponderar os valores dos indicadores específicos ideais através da seguinte tabela:

Tabela 2: Classificação dos Indicadores de Salubridade. 


\begin{tabular}{cc}
\hline Insalubre & $0-25,50$ \\
Baixa Salubridade & $25,51-50,50$ \\
Média Salubridade & $50,51-75,50$ \\
Salubre & $75,51-100,00$ \\
\hline
\end{tabular}

Fonte: BATISTA, 2005.

O presente estudo foi realizado no município de São Pedro do Iguaçu, localizado na região Oeste do estado do Paraná - Brasil, a qual consiste de uma população com cerca de 6.486 habitantes, englobando uma área com de aproximadamente $308.324 \mathrm{~km}^{2}$, Toderke (2009), é cercada por floresta estacional semidecidual, um tipo de vegetação pertencente ao bioma da Mata atlântica que abrange uma área protegida, o Parque Estadual Cabeça do Cachorro. Todos os dados coletados foram obtidos por meio de ofícios e questionários destinados aos órgãos responsáveis pelas áreas de saneamento ambiental.

\section{RESULTADO E DISCUSSÃO}

A partir do estudo, do município descrito, foi possível a montagem de uma tabela que mostra os cálculos dos resultados finais.

Tabela 3: Resultados dos indicadores de salubridade.

\begin{tabular}{lllllll}
\hline $\mathrm{I}_{\mathrm{AB}}$ & $\mathrm{I}_{\mathrm{ES}}$ & $\mathrm{I}_{\mathrm{RS}}$ & $\mathrm{I}_{\mathrm{CV}}$ & $\mathrm{I}_{\mathrm{RH}}$ & $\mathrm{I}_{\mathrm{SA}}$ & Resultado \\
100 & 80 & 60 & 81,25 & 75 & 79,58 & Salubre \\
\hline
\end{tabular}

Analisando os resultados observa-se que o município em questão possui um indicador salubre, condição ideal para os moradores da cidade, partindo do ponto que todo o resultado encontrado foi inserido a fórmula para gerar o ISA e assim analisado por Batista 2005.

$\mathrm{O}$ indicador de abastecimento de água contém um valor alto, considerando o fato de a empresa atender os requisitos de qualidade definidos anteriormente, como o bom atendimento à demanda local e tratamento da água, conseguindo assim, atender toda a população local.

Referindo-se ao indicador de esgoto sanitário o município não contam com redes coletoras apenas tanques sépticos, que de fato fez com que o valor encontrado não fosse superior. 
Já o indicador de resíduos foi inferior à média encontrada dos outros valores devido à grande demanda populacional urbana o que acarretou a falta da coleta de lixo na área rural, porém houve um correto tratamento do material coletado.

$\mathrm{O}$ indicador de controle de vetores obteve um valor superior à média, valor pelo fato de não ter ocorrências no município de leptospirose, transmissão feita pelo rato e esquistossomose, perpetuada através de uma espécie de caramujo.

Indicador de controle de recursos hídricos obteve um valor relevante referente à água bruta e disponibilidade dos mananciais, e, a empresa que dispõe do controle aquífero, afirmou que aproximadamente 340 imóveis possuem poços artesianos, o que pode estimular a utilização de água por parte da população.

Assim, observa-se que a necessidade de buscarem-se soluções é reduzida, devem-se manter as ações efetuadas, e se possível elaborar alguns projetos novos para melhorar os indicadores, além de investir em redes de esgotos para não sobrecarregar os tanques sépticos existentes, e solucionar-se a questão da coleta de lixo na zona rural assim haverá um melhoramento no índice geral.

\section{CONCLUSÃO}

Este estudo buscou o levantamento e a análise dos índices de salubridade ambiental do município de São Pedro do Iguaçu. A partir dos dados levantados chegou-se a um valor de 79,58 confirmando o desenvolvimento salubre da cidade.

Apesar de expor uma deficiência no setor sanitário, e aparentemente uma preocupação menor com a situação da coleta do lixo, o município pode adotar algumas medidas como investimento em suas redes e coleta na zona rural, podendo investir na compra de novos caminhões e contratação de empregados. Assim, no geral, a cidade vem se desenvolvendo com sucesso devendo assim, manter as iniciativas já adotadas como o tratamento correto da água, a coleta dos resíduos realizada periodicamente e o controle dos vetores, permite um desenvolvimento sustentável municipal. 


\section{REFERÊNCIAS}

ARAVÉCHIA JÚNIOR, José Carlos. Indicador de Salubridade Ambiental (ISA) para a região Centro-Oeste: Um estudo de caso no Estado de Goiás. 2010. 19 p. Dissertação (Mestrado) - Curso de Planejamento e Gestão Ambiental, Universidade Católica de Brasília, Brasília - $\quad$ Df, 2010.2 Disponível em: <http://www.bdtd.ucb.br/tede/tde_busca/arquivo.php?codArquivo=1301>. Acesso em: 27 fev. 2014.

BAHIA, Jorge Augusto. A aplicação do Indicador de Salubridade Ambiental (ISA) na determinação da vulnerabilidade dos recursos hídricos superficiais da bacia hidrográfica do Rio Cachoeira - Sul da Bahia. 2006. 30 p. Dissertação (Mestrado) - Curso de Desenvolvimento Regional e Meio Ambiente, Uesc, Ilhéus - Bahia, 2006. Disponível em: <http://www.uesc.br/cursos/pos_graduacao/mestrado/mdrma/teses/dissertacao_jorge_bahia.p df>. Acesso em: 05 mar. 2014.

BATISTA, Marie Eugénie Malzac. Desenvolvimento de um sistema de apoio à decisão para gestão urbana baseado em indicadores ambientais. 2005. 1-6 p. Dissertação (Mestrado) Curso de Pós-graduação em Engenharia Urbana, Universidade Federal da Paraíba, João Pessoa PB, $2005 . \quad$ Disponível em: <http://www.ct.ufpb.br/pos/ppgecam/images/arquivos/dissertacoes/2003/09-2003.pdf〉. Acesso em: 8 mar. 2014.

FIOROTTI, Luis. Compreenda o Saneamento Ambiental. Presidente do Crea - Es: Creaes, 2008. 6-7 p. Disponível em: <http://www.creaes.org.br/creaes/Portals/0/Documentos/cartilhas/Cartilha_Saneamento_Amb iental.pdf>. Acesso em: 23 mar. 2014.

PIZA, F. J. T. Indicador de salubridade ambiental. Seminário sobre indicadores de Sustentabilidade. São Paulo. Anais, 2000. Disponível em: <http://www.nepo.unicamp.br/textos/publicacoes/livros/migracao_urbanas/02pronex_17_Indi cador_de_Salubridade_Ambiental.pdf>. Aceso em: 09 mar. 2014.

THAME, Antonio Carlos Mendes. Indicador de Salubridade Ambiental - Manual Básico. São Paulo - SP: Sabesp, 1999. 5-7 p.

TODERKE, Marlene Lívia. Composição Florística Arbórea do Parque Estadual Cabeça do Cachorro, São Pedro do Iguaçu, Paraná. 2009. 2 p. TCC (Graduação) - Curso de Ciências Biológicas, Universidade Católica do Paraná, Toledo - Pr, 2009. Disponível em: $<$ https://www.google.com.br/search?q=sao+pedro+do+iguaçu\&oq=sao+pedro+do+iguaçu\&a 
$\mathrm{qs}=$ chrome..69i57j015.3160j0j8\&sourceid=chrome \&espv=210\&es_sm=93\&ie=UTF8\#q=sao+pedro+do+iguaçu+bioma>. Acesso em: 11 mar. 2014 